\title{
Ghrelin promotes thymopoiesis during aging
}

\author{
Vishwa Deep Dixit, ${ }^{1,2}$ Hyunwon Yang, ${ }^{1,2}$ Yuxiang Sun, ${ }^{3}$ Ashani T. Weeraratna, ${ }^{1}$ \\ Yun-Hee Youm, ${ }^{2}$ Roy G. Smith, ${ }^{3}$ and Dennis D. Taub ${ }^{1}$ \\ ${ }^{1}$ Clinical Immunology Section, Laboratory of Immunology, Intramural Research Program, National Institute on Aging, \\ $\mathrm{NIH}$, Baltimore, Maryland, USA. 'Laboratory of Neuroendocrine Immunology, Pennington Biomedical Research Center, Louisiana State University, \\ Baton Rouge, Louisiana, USA. ${ }^{3}$ Roy M. and Phyllis Gough Huffington Center on Aging, Baylor College of Medicine, Houston, Texas, USA.
}

\begin{abstract}
The decline in adaptive immunity, $T$ lymphocyte output, and the contraction of the TCR repertoire with age is largely attributable to thymic involution. The loss of thymic function with age may be due to diminished numbers of progenitors and the loss of critical cytokines and hormones from the thymic microenvironment. We have previously demonstrated that the orexigenic hormone ghrelin is expressed by immune cells and regulates $T$ cell activation and inflammation. Here we report that ghrelin and ghrelin receptor expression within the thymus diminished with progressive aging. Infusion of ghrelin into 14-month-old mice significantly improved the age-associated changes in thymic architecture and thymocyte numbers, increasing recent thymic emigrants and improving TCR diversity of peripheral T cell subsets. Ghrelin-induced thymopoiesis during aging was associated with enhanced early thymocyte progenitors and bone marrow-derived $\mathrm{Lin}^{-} \mathrm{Sca}^{+}{ }^{+} \mathrm{CKit}^{+}$cells, while ghrelin- and growth hormone secretagogue receptor-deficient (GHS-R-deficient) mice displayed enhanced age-associated thymic involution. Leptin also enhanced thymopoiesis in aged but not young mice. Our findings demonstrate what we believe to be a novel role for ghrelin and its receptor in thymic biology and suggest a possible therapeutic benefit of harnessing this pathway in the reconstitution of thymic function in immunocompromised subjects.
\end{abstract}

\section{Introduction}

The thymus is critical for the development, selection, and maintenance of the peripheral $\mathrm{T}$ cell pool possessing a broad spectrum of TCR specificities. The mammalian thymus is capable of generating $\mathrm{T}$ cells throughout the life span. However, after puberty and with advancing age, the thymic space becomes progressively filled with adipocytes coupled with a dramatic loss of thymocytes in the cortical and medullary areas, leading to a reduction in output of naive T cells. This process is called as thymic involution (1).

The lack of a thymus in humans (DiGeorge syndrome) and thymectomy of neonatal mice lead to severe immunodeficiency due to paucity of mature T cells. During physiological aging, the total peripheral $\mathrm{T}$ cell pool is maintained by homeostatic expansion of preexisting $T$ cells rather than replenishment by thymic export (2). Consequently, the long-lived naive $T$ cell repertoire is reduced with an expansion of memory phenotype $\mathrm{T}$ cells, thereby limiting a host's ability to mount responses against new antigenic challenges. It has been demonstrated that defects in naive $\mathrm{CD}^{+} \mathrm{T}$ cells are also due to the chronologic age of the naive cells themselves rather than the chronologic age of the host (3). Involution of the thymus with age and lack or paucity of newly formed naive $\mathrm{CD} 4^{+} \mathrm{T}$ cells is therefore believed to be responsible for much of the deterioration in adaptive immunity and the resultant immune dysfunction in the elderly (4). However, several recent studies have demonstrated that the old thymus still retains the capacity for $\mathrm{T}$ cell lymphopoiesis with the ability to mount functional immune responses, albeit to a limited extent $(3,5)$. Thus restoration of thymic func-

Nonstandard abbreviations used: CDR3, complementarity-determining region 3; CLP, common lymphoid progenitor; CMJ, corticomedullary junction; DN, double negative; DP, double positive; ETP, early thymocyte progenitor; GH, growth hormone; GHS-R, GH secretagogue receptor; Ker5, keratin 5; KGF, keratinocyte growth factor; LSK, Lin-Sca $^{+}{ }^{+} \mathrm{Kit}^{+}$; RTE, recent thymic emigrant; SP, single positive; TEC, thymic epithelial cell; TREC, TCR excision circle; $\mathrm{V} \beta$, variable region $\beta$.

Conflict of interest: The authors have declared that no conflict of interest exists. Citation for this article: J. Clin. Invest. 117:2778-2790 (2007). doi:10.1172/JCI30248. tion and $\mathrm{T}$ cell export in the aged host remains a promising therapeutic goal. Recent studies have demonstrated that thymus lacks a pool of self-renewing progenitors and needs continuous seeding of lymphoid progenitors from the bone marrow (6). Accordingly, it has been elegantly demonstrated that early thymocyte progenitors (ETPs) decline markedly with age and that these progenitors have an increased rate of apoptosis with reduced proliferative capacity (7). The precise mechanisms responsible for thymic involution with age remain to be identified and presumably involve complex multisystem interactions, wherein various cytokines, neuropeptides, and hormones influence immune compartment.

Ghrelin is a peptide hormone predominantly produced by the $\mathrm{X} / \mathrm{A}$-like enteroendocrine cells (a major endocrine population of ovoid cells with compact electron-dense granules that are present in the oxyntic mucosa of rodents and humans) in the stomach in response to negative energy balance and stimulates hunger and promotes growth hormone $(\mathrm{GH})$ secretion from the pituitary via binding to its 7 transmembrane $G$ protein-coupled receptor, the GH secretagogue receptor (GHS-R) $(8,9)$. Ghrelin is acylated on its third serine residue, and this octanoylation is critical for binding to the GHS-R and the biological activities of ghrelin (10). The ghrelin receptors were traditionally thought be highly expressed in the pituitary and the CNS, but recent studies have identified GHS-Rs in various cells and organs systems including immune cells $(11,12)$. Ghrelin and GHS-R are expressed by peripheral resting and activated human T cells (12) and have been shown to mediate antiinflammatory signals in several cells types $(13,14)$, including mononuclear cells $(12,15)$, and in various murine models of acute and chronic inflammation (13-17). Involution of thymus is accompanied by replacement of the $T$ cell compartment via infiltration of adipocytes and an increase in proinflammatory cytokines such as IL-6 within the thymic microenvironment (18). Given the ability of ghrelin to inhibit proinflammatory cytokines from immune cells and its capacity to stimulate GH-IGF-1 axis, we hypothesized a role for ghrelin and its receptor in age-related 

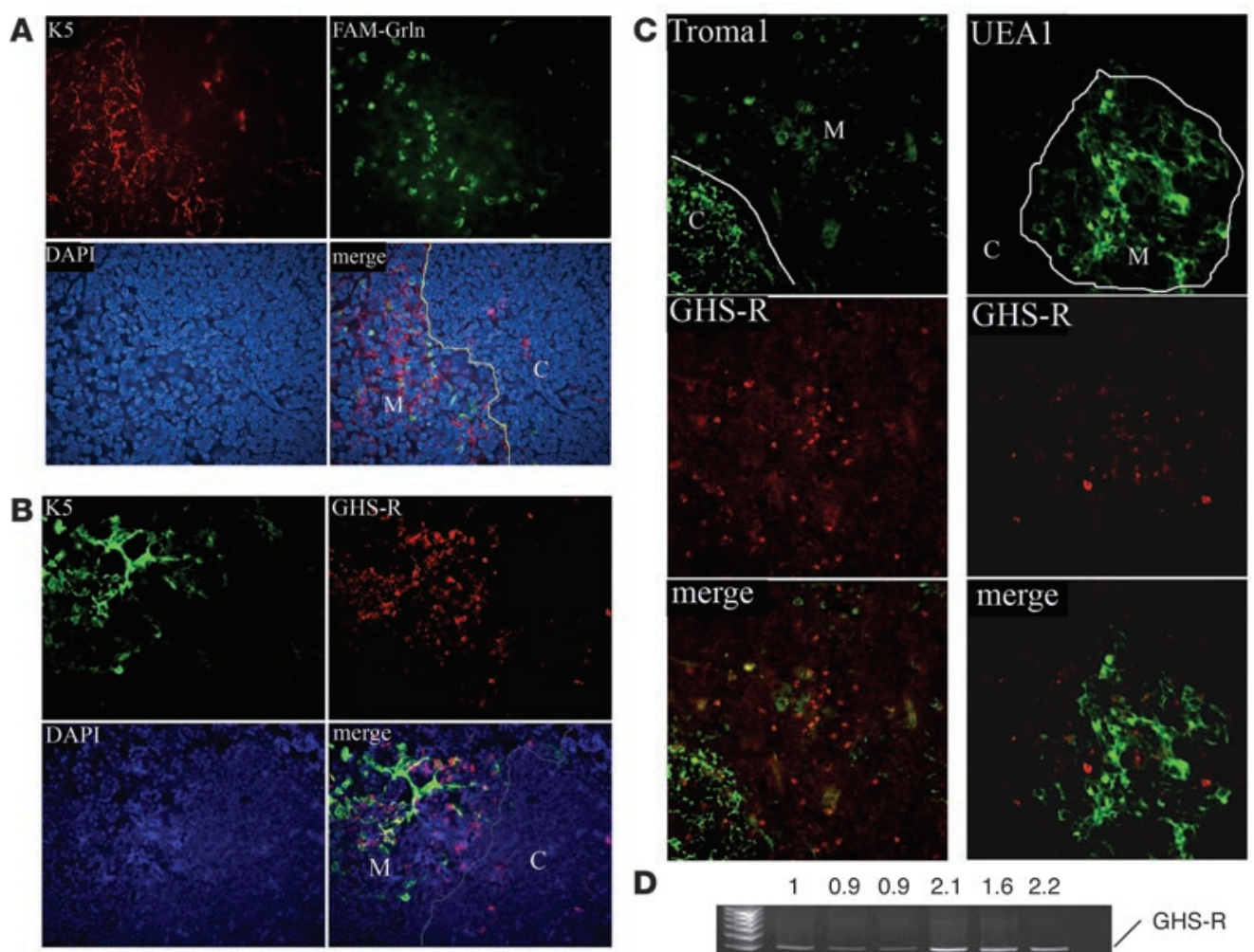

D

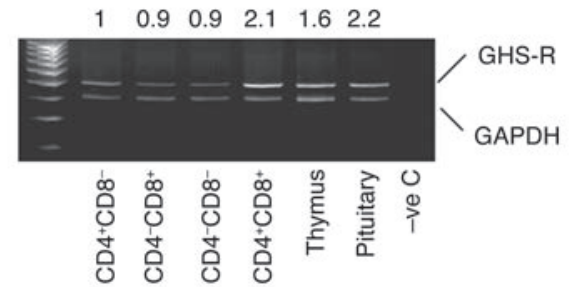

Figure 1

The GHS-R expression in thymus. (A) The ghrelin binding sites exist largely in the medullary area of the thymus. Frozen thymic sections were labeled with FAM-conjugated ghrelin (FAM-Grin) and stained with medullary epithelial cell marker, Alexa Fluor 594-conjugated anti-Ker5 (K5) antibody and counter-stained with DAPI for nuclei. Ghrelin binding sites (green) are largely segregated from Ker5-expressing cells in the cortex (C; red), as shown by lack of a yellow staining pattern, which would suggest colocalization (line depicts the $\mathrm{CMJ}$ in the thymus). M, medulla. (B) Similar to FAM-ghrelin binding, the GHS-R antibody-positive cells displayed only modest colocalization with Ker5 $5^{+}$medullary TECs. (C) GHS-R (red) displayed little to no colocalization with cortical Ker8 ${ }^{+}$and medullary UEA-1+ TECs. However, a minor subset of Ker8 ${ }^{+}$cells in the medulla displayed GHS-R immunopositivity. (D) Thymus and sorted SP, DP, and DN thymocytes express GHS-R mRNA. DP cells expressed 2-fold higher GHS-R than did SP and DN cells, with levels comparable to those of the pituitary. Numbers above the blot indicate the ratio of GHS-R to GAPDH expression. -ve C, negative control. Original magnification, $\times 10$.

\section{Results}

Ghrelin receptor expression in thymus. Initial studies focused on examining the expression of GHS-R in the thymus and on developing $\mathrm{T}$ cells. The frozen thymic sections from 2-month-old $\mathrm{BALB} / \mathrm{c}$ mice were labeled with FAM-conjugated ghrelin peptide to localize the ghrelin binding sites in the thymus. The majority of ghrelin binding sites were found to be present in the thymic medulla (Figure 1A) and displayed minor colocalization with the medullary epithelial cell marker keratin 5 (Ker5) (Figure 1A) $(29,30)$. We next confirmed the medullary localization of ghrelin receptor using the GHS-R1a antibody. Similar to our findings using FAM-labeled ghrelin, a higher GHS-R expression level in thymic medulla was observed and the majority of GHS-R was found not to be expressed in $\mathrm{Ker}^{+}$thymic epithelial cells (TECs) (Figure 1B). In order to identify whether GHS-R was expressed in other TEC subsets, the frozen thymic sections of 2-month-old C57BL/6 mice were stained for Ker8 (Troma1) and UEA-1. As expected, the majority of $\mathrm{Ker}^{+} \mathrm{TECs}$ were present in the thymic cortex and the UEA-1+ TECs were present in the thymic medullary region. While the majorthymic involution. Several potential and promising strategies for thymic reconstitution associated with aging, HIV infection, myeloablative chemotherapy, and radiation have been studied and proposed (19). In addition, recent studies have demonstrated that autologous peripheral blood stem cell transplants in breast cancer patients (20), androgen ablation by castration in old mice (21), and gonadotropin-releasing hormone agonist treatment in both rodents $(22,23)$ and prostate cancer patients (24) can significantly promote thymic renewal. In addition, past studies have demonstrated a thymopoietic and hematopoietic effects for GH $(25,26)$, IGF-1 (27), and GH secretagogues (28) during aging. Here we provide evidence that signaling of the orexigenic peptide hormone ghrelin through GHS-R serves an important biological role in generation of naive $T$ cells and ageassociated thymic involution. ity of the Ker8 positivity did not colocalize with GHS-R in the cortex, a subset of $\mathrm{Ker}^{+}$mTECs did appear to coexpress GHS-R (Figure 1C). Moreover, we failed to demonstrate any GHS-R expression on UEA-1 $1^{+}$mTECs (Figure 1C).

Ghrelin is currently known to bind specifically to GHS-R (8-10). In order to determine the GHS-R expression in T cell subsets, various $T$ cell populations were sorted and analyzed for GHS-R mRNA expression by regular RT-PCR (Figure 1D) and real-time quantitative PCR (Supplemental Figure 1; supplemental material available online with this article; doi:10.1172/JCI30248DS1). The GHS-R was found to be expressed in the thymus and on the various thymocyte subsets with highest expression in $\mathrm{CD}^{+} \mathrm{CD}^{+}$cells compared with single-positive (SP) and doublenegative (DN) cells (Figure 1D and Supplemental Figure 1A). These data suggest that GHS-Rs are largely expressed in the 

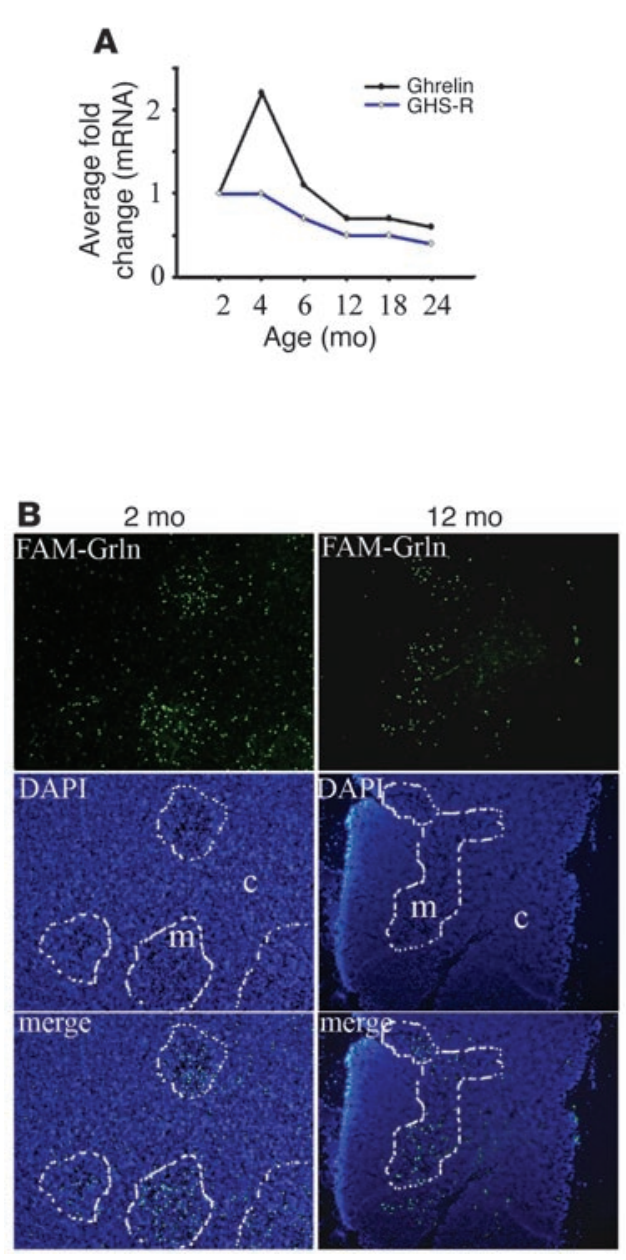

\section{C}

2 mo

$4 \mathrm{mo}$
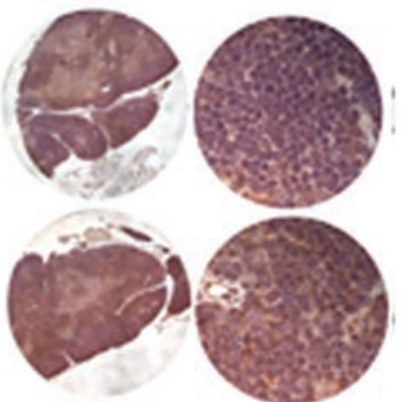

$12 \mathrm{mo}$
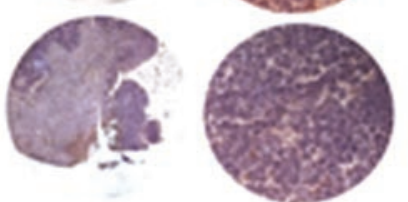

$18 \mathrm{mo}$
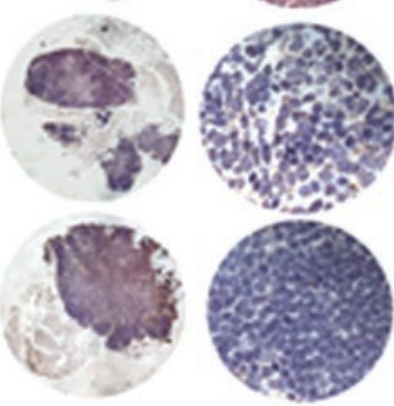

D

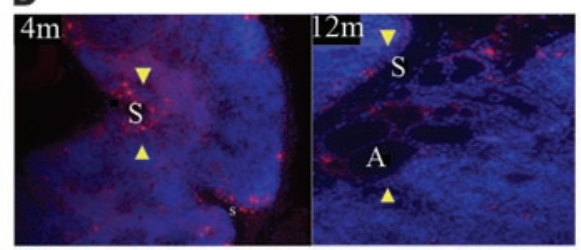

\section{Figure 2}

Ghrelin expression declines with age in thymus. (A) Real-time PCR analysis of mice $(n=5-6)$ at various ages revealed an age-dependent reduction in ghrelin and GHS-R mRNA expression. Threshold cycle values from individual animals $(n=6)$ in each group were collapsed and normalized with GAPDH and expressed as average fold change in comparison with 2-month-old mice. (B) The GHS-R expression revealed by FAM-ghrelin binding displayed primarily medullary GHS-R expression, which appears to decrease with age. Medullary regions are outlined with white lines. (C) The total ghrelin protein expression was studied in thymus of 2 , 4, 12, 18, and 24-month-old mice. Each section is representative of $4-5$ thymi in each group. High ghrelin immunopositivity (brown staining) was observed in cortex as well as medulla of 2- to 4month-old mice and declined in aging thymus. Original magnification, $\times 10$ (left); $\times 40$ (right). (D) Acylated ghrelin protein expression (arrowheads) also declines with age. Note the significant increase in large lipid vacuoles of adipocytes (A) in the septa (S) of thymus of 12-month-old mice. In contrast to 12-month-old mice, ghrelin expression was expressed in thymic cortex and the medulla in 4-month-old mice. Original magnification, $\times 10$ (B and D). developing thymocytes and that Ker5-, Ker8-, and UEA-1expressing subsets of epithelial cells may not be the primary sites of ghrelin's action within the thymus.

Ghrelin and GHS-R expression decline with age in the thymus. Given that ghrelin is expressed and secreted by T cells (12) and that the presence of growth factors in the thymic microenvironment is believed to play a critical role in $\mathrm{T}$ cell development (30), we next examined the expression of ghrelin and GHS-R in the thymus of mice of various ages. In comparison with 2-month-old mice, we observed an increase in the expression of thymic ghrelin mRNA in 4-month-old animals, followed by a subsequent decline in both ghrelin and GHS-R expression with progressive thymic aging (Figure 2A). To confirm these observations, we examined the expression of GHS-R and acylated (active) ghrelin protein in thymi of mice of varying ages. Similar to our results shown in Figure 1, we observed ghrelin binding sites to be present in the thymic medulla with an observable decrease in expression in aging mice (Figure 2B). Total ghrelin (Figure 2C) and bioactive acylated ghrelin (Figure 2D) protein expression in the thymus also displayed a dramatic reduction by 12 months of age in thymus (Figure 2C). This loss of expression strongly correlated with the thymic involution and an increase in adipocyte infiltration.

Ghrelin infusion partly reverses age-associated thymic involution. Intrathymic loss of cytokines such as IL-7 (31) and increased expression of IL- 6 and cytokines of the GP130 family (18) with age have also been hypothesized to contribute to age-associated thymic involution. The loss of ghrelin and ghrelin receptor with aging might similarly contribute to the involution process. Therefore additional studies were aimed at assessing the ability of acylated ghrelin to reconstitute thymic function in aged mice. Ghrelin infusion into 14 -month-old mice via s.c. osmotic mini-pumps over a 2 -week time period led to a significant increase in thymic mass (Figure $3 \mathrm{~A}$ ) and a greater than 2 -fold increase in thymocyte numbers in 14-, 20-, and 24-month-old but not in 2-monthold BALB/c mice (Figure 3B). Similar pro-thymic effects were observed in 8-month-old C57BL/6 mice upon ghrelin infusion (Supplemental Figure 1C), suggesting that effects of ghrelin on thymic cellularity are not strain dependent. It has been previously reported that activation of the GH-IGF-1 axis can significantly attenuate thymic involution (25) and promote hematopoiesis (26). Ghrelin is also known to be a potent stimulator of the somatotropic axis in the young animals (8); therefore, we next examined the impact of ghrelin infusion on the GH-IGF-1 axis in old mice. While we observed a significant increase in body weight in ghrelin-infused 14-month-old mice (Figure 3C) and an increase in GH immunopositivity in the anterior pituitary (Supplemental Figure 1B), we did not detect significant differences in the serum IGF-1 levels (Figure 3D) or IGF-1 mRNA expression in liver (data not shown) upon ghrelin treatment. As GH is pulsatile 
A

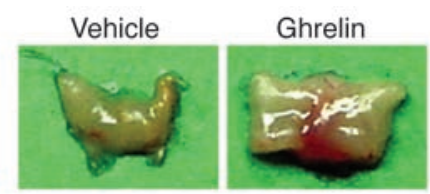

B

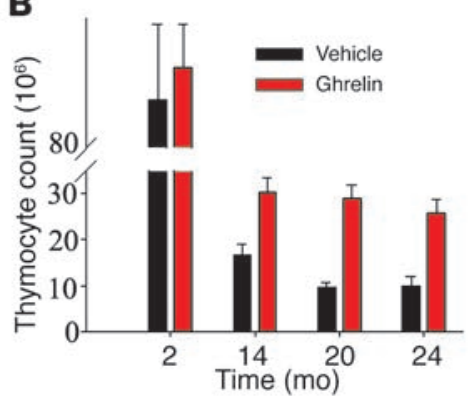

F

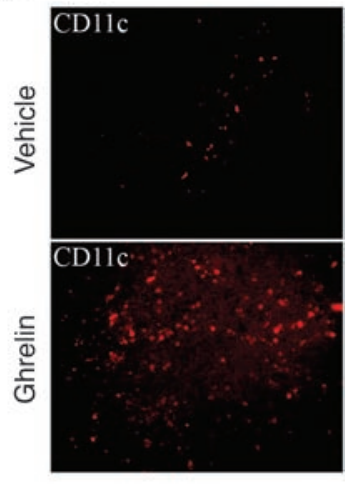

C

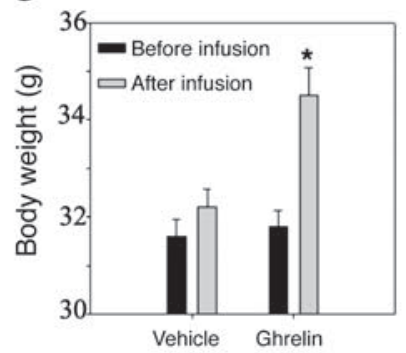

D

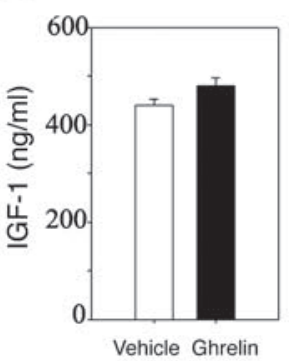

E

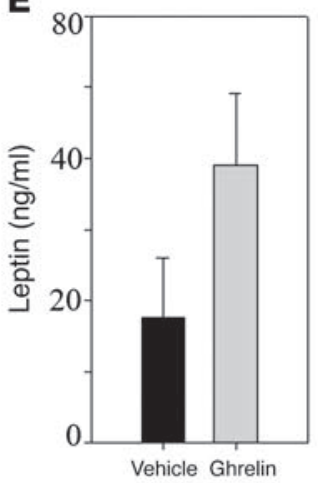

G
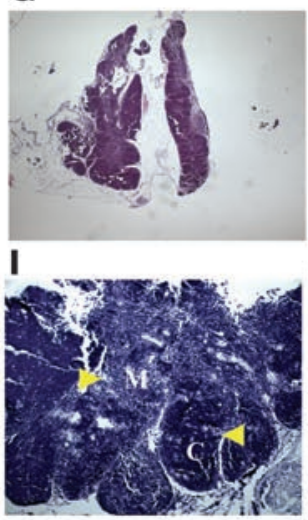

K

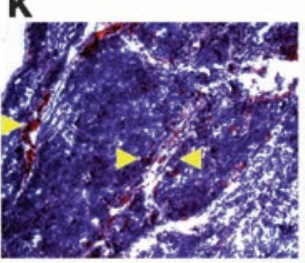

H

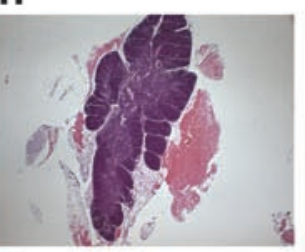

J

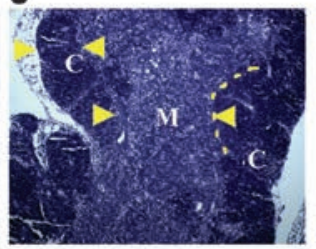

L

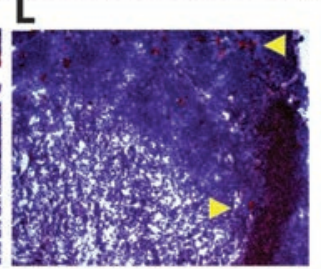

\section{Figure 3}

Ghrelin enhances thymic size and cellularity in aging mice. (A) Ghrelin infusion for 2 weeks via s.c. osmotic pumps caused a significant increase in thymic size in 14-month-old animals. (B) Ghrelin did not alter thymocyte counts in the 2-month-old mice but significantly increased total thymocyte numbers in 14-, 20- and 24-month-old mice. (C-E) Ghrelin treatment significantly increased body weight in 14-month-old mice (C) without a significant change the peripheral IGF-1 (D) or leptin (E) levels. ${ }^{*} P<0.05$. (F) Ghrelin infusion into 14-month-old mice led to increased GHS-R expression (green) with partial colocalization with CD11 $\mathrm{C}^{+}$cells. In addition, ghrelin administration resulted in an enhanced number of CD11 $\mathrm{c}^{+}$ dendritic cells in the thymic medulla. (G-J) Compared with 14-month-old vehicle-infused animals, ghrelin infusion significantly improved the thymic architecture. Ghrelin infusion is associated with increased cellularity, clear delineation (arrowheads) of cortex (dark stain) from medulla (light stain) and well defined CMJ (dotted line), the site where progenitors arrive in thymus. (K and $\mathbf{L})$ Frozen thymi from 14-month-old mice stained for Oil Red O displayed an increased number of "adipocyte-like" lipid-laden cells (arrowheads) in the parenchyma and perivascular space, while in ghrelin-infused mice, a marked reduction in PVS (K and $\mathbf{L})$ with reduced numbers of lipid droplet-containing cells $(\mathbf{L})$. Original magnification, $\times 5(\mathbf{G}$ and $\mathbf{H}) ; \times 10(\mathbf{F}, \mathbf{I}$, and $\mathbf{J}) ; \times 20(\mathbf{K}$ and $\mathbf{L})$.

in nature, IGF-1 is often utilized as a surrogate marker of GH activity. These data suggest that GH may not be playing a major role in the observed responses.

We have previously reported that ghrelin inhibits leptin-induced proinflammatory cytokine expression in vitro and in vivo (12). To determine whether there is any possible interaction by leptin in the pro-thymic effects of ghrelin, we also analyzed the circulating leptin levels in vehicle- and ghrelin-infused mice. Interestingly, we observed that ghrelin supplementation in 14-month-old mice $(n=15)$ led to a modest increase in serum leptin levels (Figure 3E); however, this increase did not reach statistical significance due to individual variations in the mice. However, given that leptin-defi- cient $o b / o b$ mice have highly involuted thymus (32) and leptin can attenuate LPS-induced thymic involution (33), it is possible that leptin-ghrelin interactions may play some role in thymopoiesis.

Histological examination of thymus from ghrelin-infused 14month-old mice revealed marked improvement of the thymic architecture following treatment. Typically the ghrelin-infused thymi display an increase in cortical and medullary cellularity along with well defined corticomedullary junctions (CMJs) when compared with vehicle-infused thymi (Figure 3, G-J). It has been suggested that increased infiltration by lipid-bearing adipocytelike cells correlate with loss of thymic cellularity and involution (1). We observed a marked reduction in lipid droplet-contain- 

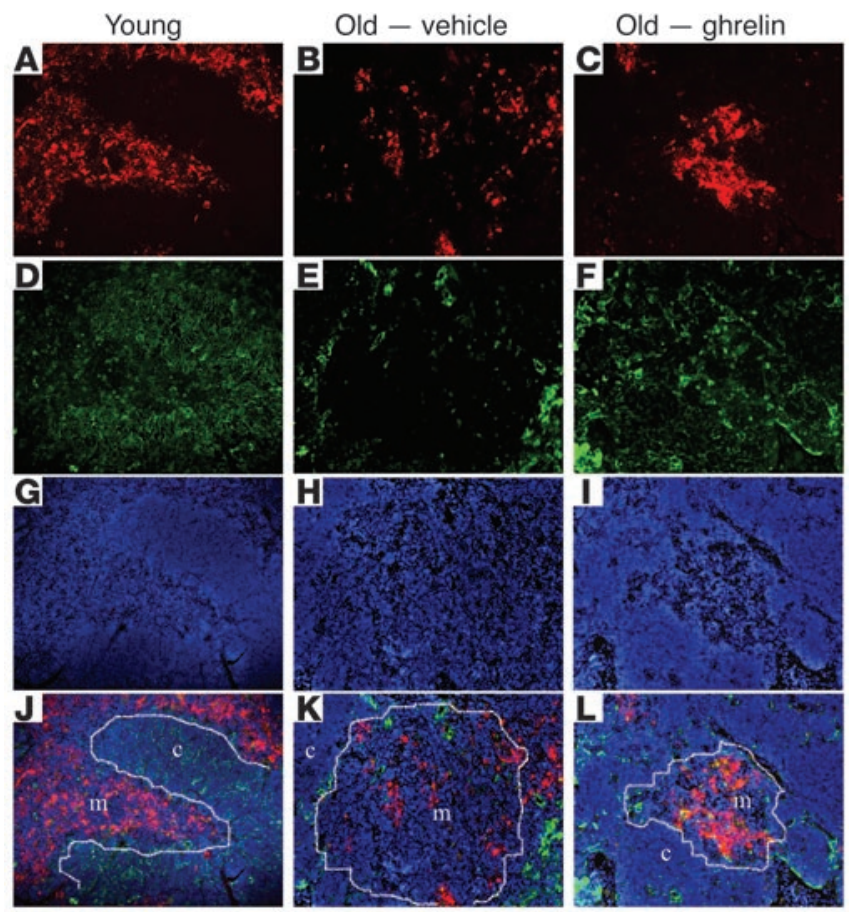

Figure 4

Ghrelin renews the TEC compartment during aging. The thymus from young mice displays abundant UEA-1+ ${ }^{+}$cells $\left(\mathbf{A}-\mathbf{C}\right.$; red) and Ker $8^{+}$cells (D-F; green) in the medulla and cortex. (B, E, H, and K) The aging thymus of 14-month-old mice displayed diffuse and reduced medullary UEA $-1^{+}$cells and Ker8+ cortical TECs. (C, F, I, and L) However, compared with vehicle-treated aged mice, ghrelin infusion resulted in a significant increase in both medullary and cortical TECs based on the increase in UEA-1 and Ker8 staining. (G-I) DAPI staining to visualize the nuclei of the cells. (J-L) Combined images of the upper panels. Yellow staining denotes colocalization. Original magnification, $\times 10$.

ing cells in the thymic perivascular space of 14-month-old mice infused with ghrelin (Figure 3, $\mathrm{K}$ and $\mathrm{L}$ ). A reduction in adipocyte deposition in ghrelin-infused mice may facilitate the generation of $T$ cells by preserving the thymic architecture and improving the aged thymic microenvironment.

Thymic dendritic cells present self-antigens to developing thymocytes, play a crucial role in central tolerance induction, and facilitate the process of negative selection. We therefore examined whether the thymic improvements associated with ghrelin infusion led to a change in the numbers of thymic dendritic cells. Compared with vehicle-infused 14-month-old mice, we did observe a significant increase in the CD $11 \mathrm{c}^{+}$dendritic cells in the aged thymi of ghrelin-infused animals (Figure 3F). Interestingly, the doublelabeling experiments revealed that ghrelin infusions also increased medullary GHS-R expression, which displayed a partial colocalization with CD11c cells (Figure 3F).

It has recently been suggested that cortical and medullary TECs have potent capacity to stimulate naive $T$ cells (comparable with the capacity of thymic dendritic cells) and that age-associated thymic involution is associated with aberrations in the TEC compartment $(30,34)$. Analysis of UEA- $1^{+}$medullary and $\mathrm{Ker} 8^{+}$cortical TECs revealed a marked reduction in both cortical and medullary TECs in the aging thymus (Figure 4, B, E, and K). Interestingly, ghrelin infusion into 14-month-old animals led to a significant increase in $\mathrm{Ker}^{+}$cortical TECs (Figure 4, E and F). In addition, compared with diffused and reduced UEA-1 positivity in thymic medulla of 14-month-old mice, we observed that ghrelin infusion also led to an increase in the numbers of UEA $-1^{+}$medullary TECs compared with vehicle-infused mice (Figure 4, B, C, E, F, H, I, K, and L) without significant changes in Aire mRNA expression (Supplemental Figure 2). Collectively these data suggest that ghrelin significantly restores the age-associated decline in the TECs within the thymus.

Ghrelin promotes generation of recent thymic emigrants with a diverse $T C R$ repertoire. The $\mathrm{T}$ cell output and generation of newly formed $\mathrm{T}$ cells from the thymus can be evaluated by quantifying the presence of TCR excision circles (TRECs) in peripheral T cells that have not proliferated after rearrangement of their TCR (35). Given that ghrelin induces an increase in thymic cellularity and improvement of thymic architecture in aging mice, we next quantified thymopoiesis and recent thymic emigrants (RTEs) by measuring the excisional DNA of TCR gene rearrangement. Compared with control animals, we observed that ghrelin infusion increased the levels of signal-joint TRECs in purified splenic $T$ cells by greater than 2 -fold, suggesting increased thymopoiesis in 14-month-old animals (Figure 5A). Given that both aging and decline in thymic output compromise the $\mathrm{T}$ cell diversity (36), we next analyzed the effects of ghrelin infusion on TCR diversity through the use of complementarity-determining region 3 (CDR3) length polymorphism analyses (37). The exquisite antigen specificity of TCR is largely dependent on the hypervariable CDR 3 region of the $\beta$ chain. Analysis of peripheral $C D 4^{+}$and $C D 8^{+}$ $\mathrm{T}$ cells from ghrelin-infused mice (Supplemental Figures 3 and 4) revealed a significant improvement in $V \beta$ spectral profiles. More specifically, we observed significant improvement in the diversity of $\mathrm{V} \beta 8.1,-8.2$, and -8.3 (Figure 5, B and C) in total peripheral T cells as well as in $\mathrm{CD}^{+}$and $\mathrm{CD}^{+}$subsets and additionally V $\beta 4,-16$, and -19 in the $\mathrm{CD}^{+} \mathrm{T}$ cell subset (Figure 5, C and D). Thus the emergence of RTEs in the periphery upon ghrelin infusion was associated with an improvement of TCR diversity from an oligoclonal-like pattern to a more polyclonal Gaussian profile.

Ghrelin promotes thymopoiesis by increasing the lymphoid progenitors. It has previously been reported that the earliest thymocyte precursors (negative for CD4 and CD8) arrive at CMJ in thymus and subsequently differentiate into mature $\mathrm{T}$ cells $(30,34,38)$. The developmental progression of these progenitors can be monitored by expression of CD44 and CD25. These DN progenitors develop into CD $44^{+} \mathrm{CD} 25^{-}$DN1 cells followed by CD $44^{+} \mathrm{CD} 25^{+} \mathrm{DN} 2$, CD $44^{-}$

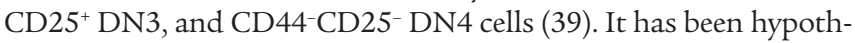
esized that there is a specific block in the transition of DN1 to DN2 stages with aging and that this is one of the possible mechanisms responsible for reduced thymopoiesis in old animals. IL-7 and castration have been reported to reverse the age-associated DN1 block (24, 40). However, recent work has revealed that the DN1 compartment is developmentally diverse and includes cells of $\mathrm{B}, \mathrm{NK}$, and myeloid lineages (6). Utilizing a broad array of cell surface markers to exclude $\mathrm{Lin}^{+}$cells, it has been observed that ETPs within the DN1 population are identified as $\mathrm{Lin}^{-} \mathrm{CD} 127^{-} \mathrm{CD} 25^{-} \mathrm{CD} 44^{+} \mathrm{CKit}{ }^{+}$. The ETPs, unlike the total DN1 cells, have been shown to decline with age (7). Recent work from Bhandoola and colleagues (41) has demonstrated that a significant number of DN1 cells express CD4, hence the lineage cocktail for ETP enumeration excluded the CD4 antibody. Furthermore, $\mathrm{Lin}^{-} \mathrm{CD} 25^{-} \mathrm{CKit}^{+}$cells in the adult thymus uniformly express high levels of CD44. Therefore, based on these studies we next analyzed the ETP, DN2, DN3, and DN4 populations in thymus of aging mice excluding the CD44 antibody from the cocktail (41). 
A

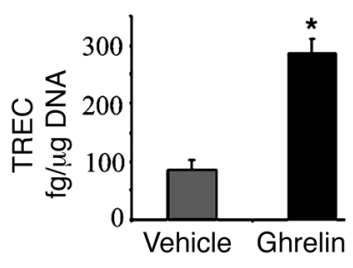

B $\quad$ V- $\beta 8.1 \quad$ V $-\beta 8.2 \quad$ V $-\beta 8.3$
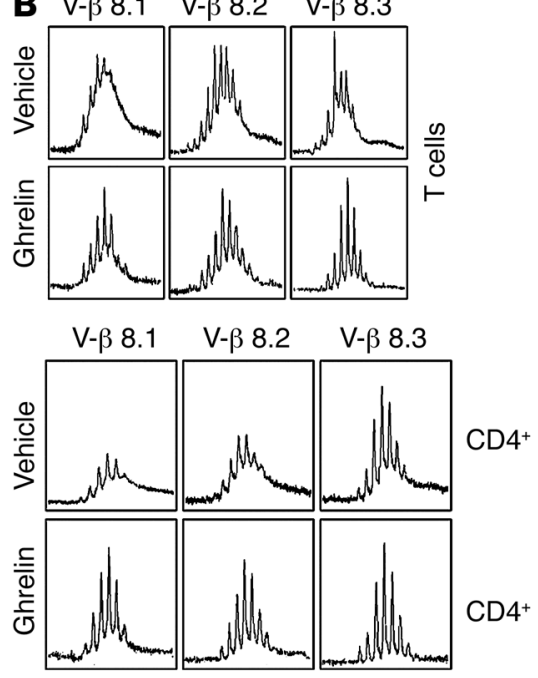

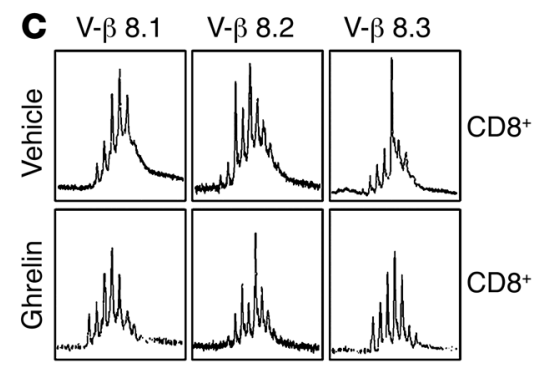

D $\quad$ V $-\beta 8.1 \quad$ V $-\beta 8.2 \quad$ V $-\beta 8.3$

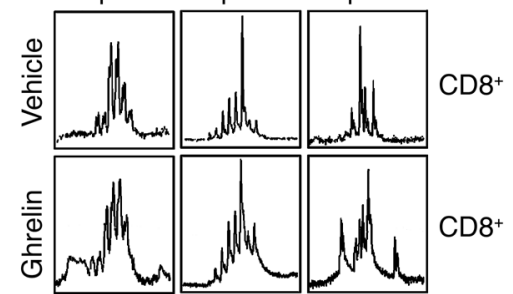

Figure 5

Ghrelin increases thymopoiesis in old animals. (A) Ghrelin increased the number of RTEs in aged mice. Equal amount of DNA (0.5 $\mu g)$ from purified ghrelin- or vehicle-infused 14-month-old BALB/c splenic T cells was analyzed for signal-joint TRECs by quantitative PCR. (B) The RNA from purified splenic T cells was isolated and pooled from 6 individual vehicle- and ghrelin-infused 14-month-old BALB/c mice and then analyzed for TCR diversity by CDR3 length PCR analysis. The immunoscopic profile of TCR V $\beta$ families with a polyclonal repertoire for a specific $V \beta$ family is represented by $6-8$ peaks arranged in a Gaussian profile, with the highest-intensity CDR3 segment in the center. (C) Ghrelintreated 14-month-old mice displayed a significant improvement of $\mathrm{V} \beta 8$ diversity in splenic $\mathrm{T}$ cells and purified CD4+ and $\mathrm{CD}^{+}$lymphocytes. CDR3 sizes are shown on the $x$ axis, and the peak fluorescence intensity is shown on the $y$ axis. (D) Ghrelin also specifically improved V $\beta 4$, -16 , and -19 polyclonality of CD8+ cells.

We have observed that ghrelin infusion induces a greater than 2-fold increase in the ETPs in 14-month-old mice (Figure 6, B and C). The increase in ETP in the DN1 compartment of aging mice following ghrelin infusion was not associated with significant changes in DN2 $\left(\mathrm{Lin}^{-} \mathrm{CKit}^{+} \mathrm{CD} 25^{+}\right.$), DN3 (Lin ${ }^{-} \mathrm{Kit}^{-} \mathrm{CD} 25^{+}$), or DN4 subpopulations. In addition, neither ghrelin nor des-acyl ghrelin affected peripheral CD4/CD8 ratios (Figure 6A). Furthermore, in addition to ETPs, the $\mathrm{DN} 1$ population is comprised of $\mathrm{Lin}^{-} \mathrm{CD} 117^{-} \mathrm{CD} 25^{-} \mathrm{CD} 44^{+} \mathrm{CD} 45 \mathrm{R}^{+}$ cells characteristic of the common lymphoid progenitor (CLP). The aging thymus lacks the self-renewing pool of thymocyte progenitors and requires seeding by bone marrow-derived hematopoietic stem cells characterized as $\mathrm{Lin}^{-} \mathrm{Sca} 1^{+} \mathrm{cKit}^{+}$(LSK) cells (6). We also observed that ghrelin-induced increase in the thymic lymphocyte progenitors was associated with a significant increase in the BM-derived LSK population in 14-month-old mice (Figure 6, D and E).

Ghrelin's regulatory counterpart, leptin, enhances thymopoiesis in aged, but notyoung, mice. We have previously demonstrated that, similar to their opposing metabolic effects on food intake, leptin and ghrelin also exert reciprocal regulatory effects on proinflammatory cytokine production (12). Ghrelin inhibits activation- and leptin-induced proinflammatory cytokine production by human $T$ cells and monocytes (12). Given the interplay between these 2 hormones, we next examined whether leptin might also influence thymopoiesis in aged mice. Similar to ghrelin, we observed that leptin infusion also significantly increased thymocyte counts (Figure 7A) and increased the RTE (Figure 7B) in aged but not young mice. However, unlike ghrelin, leptin infusion increased the peripheral IGF-1 levels (Figure 7C) and upregulated keratinocyte growth factor (KGF) expression in aging thymus (Figure 7D). In addition, the action of ghrelin and leptin on thymus appeared to be distinct, as only ghrelin specifically inhibited TNF- $\alpha$ (Figure 7F) and enhanced SCF (Figure 7E) expression in the aging thymus. We also observed that leptin, similar to ghrelin, improves thymic architecture (Figure 7G) but interestingly, unlike ghrelin, leptin was able to mediate prosurvival effects in aging thymocytes (as demonstrated by fewer annexin-positive cells compared with controls; Figure 7H). This leptin-mediated increase in thymopoiesis was also reflected in improvement of the peripheral T cell compartment as demonstrated by TCR spectratyping analysis (Figure 7I). These data suggest that both leptin and ghrelin exert pro-thymic effects in aged but not young mice, possibly through distinct mechanisms based on the differential induction of IGF-1, KGF, and SCF. A balance between these 2 metabolic hormones may be critical in the regulation of thymopoiesis.

Ghrelin-and GHS-R-deficient mice display accelerated age-associated thymic involution. In order to better understand the physiological relevance of the ghrelin-GHS-R pathway on thymic development, both ghrelin and GHS-R knockout mice were examined for any alterations in T cell development and thymic function. Compared with WT littermates, no significant differences in thymic size or cellularity were observed in the young ghrelin $/-$ and GHS-R $\mathrm{R}^{-/-}$mice (Figure 8A). However, in 24-month-old ghrelin ${ }^{-/-}$and GHS-R $\mathrm{R}^{-/}$mice, a significant reduction in thymic size and thymocyte counts (Figure 8, A and B) was observed in comparison with the WT littermate controls. There was also an increased presence of adipocytes in the thymic medulla as well as cortex of GHS-R $\mathrm{R}^{-1}$ mice (Figure 8 , B and C). Thus the knockout mice appeared to have an accelerated thymic aging phenotype. We did not observe a statistically significant difference in serum IGF-1 levels in the 24-month-old GHS-R knockout mice (Figure 8D). To ascertain whether the thymopoietic effects of ghrelin are mediated via a GHS-R-specific mechanism, the 24-monthold GHS-R knockout mice $(n=5)$ were infused with ghrelin for a 2week period. The $24-$ month-old GHS-R ${ }^{+/+}$mice treated with ghrelin 
A

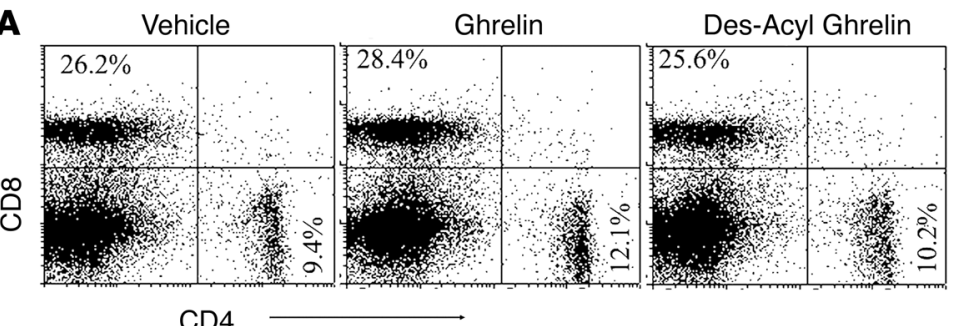

C

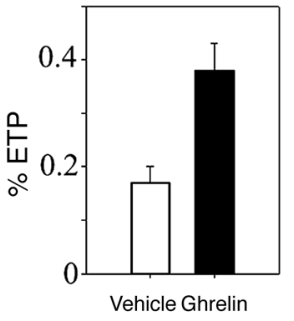

D

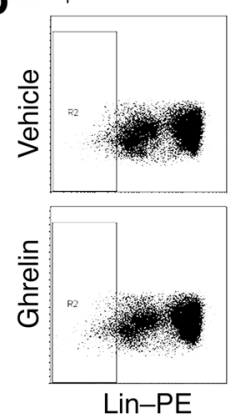

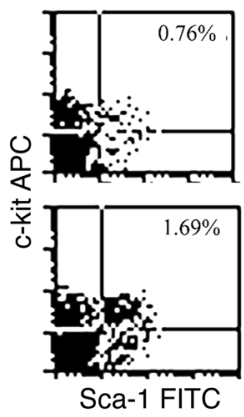

B

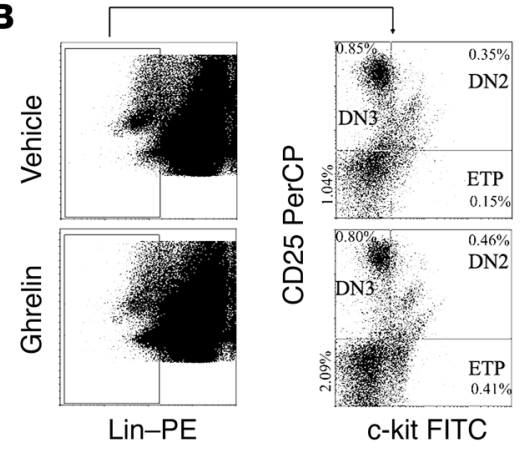

E

\section{Figure 6}

Ghrelin increases lymphoid progenitor populations in aging mice. (A) Ghrelin increased lymphoid progenitor populations in 14-month-old mice without altering the peripheral CD4/CD8 ratios. (B) The ETPs were identified as Linlow CKit ${ }^{+}$CD25-. The lineage cocktail included antibodies against CD3 $\varepsilon, C D 8 \alpha$, TCR $\alpha \beta, \gamma \delta$ TCR, NK1.1, TER119, CD11b, CD11c, CD19, CD127, and B220 but not CD4. For ETP analysis, $10^{6}$ thymocytes from 14-month-old BALB/c mice were stained for Lin-PE, CD25 PerCP, and c-kit FITC, and 700,000 total events were acquired. Lin ${ }^{\text {low }}$ cells were analyzed for c-kit and CD25 expression. (C) Ghrelin infusion in 14-month-old mice led to a 2-fold increase in ETPs in thymus $(P<0.05)$ with no significant change in DN2 ( Lin $\left.^{\text {low }} \mathrm{CKit}^{+} \mathrm{CD} 25^{+}\right)$, DN3 (Lin $\left.{ }^{\text {low }} \mathrm{CKit}-\mathrm{CD} 25^{+}\right)$, or DN4 populations. (D) Bone marrow cells were analyzed for LSK populations. (E) Ghrelin infusion significantly increased $(P<0.05)$ the BM LSK cells in 14-month-old animals. Data is represented as mean \pm SEM of 2-month-old and 14-month-old mice ( $n=6$ per group).

exhibited a significant increase in thymocyte numbers $(P<0.05)$, while the age-matched GHS- $\mathrm{R}^{-/}$mice did not show increased thymopoiesis in response to ghrelin (Figure 8E). These data suggest that ghrelin's effects on aged thymus are GHS-R specific.

Reduced thymopoiesis in absence of ghrelin signaling is associated with deficits in lymphoid progenitors and contraction of TCR repertoire of peripheral $\mathrm{CD}^{+} \mathrm{T}$ cells. Quantitation of TRECs in splenic $\mathrm{T}$ cells in the 24-month-old ghrelin-/- and GHS- $\mathrm{R}^{-/-}$mice revealed a marked reduction in the RTEs (Figure 9A). This was also reflected in a decreased number of circulating $\mathrm{T}$ cells in the blood of 14-month-old GHS-R-1- mice (Supplemental Figure 5A). The 14month-old ghrelin ${ }^{-/-}$mice did not exhibit any significant differences in the T cell development as evidenced by similar percentages of DN1, DN2, DN3, and DN4 cells (Supplemental Figure 5B). Interestingly, we observed a marked reduction of LSK cells in the bone marrow of both 24-month-old ghrelin-deficient (Figure 9B and Supplemental Figure 5C) and GHS-R-deficient animals (Figure 9B and Supplemental Figure 5D), suggesting that ghrelin might play an important role in the maintenance of the hematopoietic stem cell niche. In addition, we observed a marked perturbation in the TCR diversity of splenic $\mathrm{CD} 4^{+} \mathrm{T}$ cells derived from 24-month-old GHS-R knockout animals (Figure 9C). We also observed a striking lack of amplification of $\mathrm{V} \beta 9$ in the $\mathrm{CD} 4^{+}$ $\mathrm{T}$ cells of 24-month-old GHSR ${ }^{-/}$mice. These data clearly suggest that the ghrelin-GHS-R axis is an important physiological player in regulating thymic function during aging.

\section{Discussion}

Ghrelin is currently known to be the only endocrine hormone that stimulates food intake and meal initiation (10). However, since its discovery in 1999 (8), many functions have been attributed to ghrelin including its emerging role as an immune regulator and its antiinflammatory function in various cell types (13). The ghrelin receptors have been identified in monocytes and human $\mathrm{T}$ cells, however its potential role in age-associated thymic involution remains to be investigated. Here we provide evidence that ghrelin can significantly promote $T$ cell output from an aging thymus by promoting the recruitment of lymphoid progenitors from the bone marrow in a GHS-R-specific pathway.

Contraction of $\mathrm{T}$ cell repertoire and reduced thymic output with age is one of the primary causes of late life predisposition to various infections and suboptimal responsiveness to vaccination. Generation of naive $\mathrm{T}$ cells declines rapidly at the onset of puberty and continues to plummet with advancing age. Previous studies have demonstrated that an increase in sex steroid levels contribute to the process of thymic involution, and indeed castration can significantly augment thymic function $(21,24)$. However, during physiological aging the peripheral $\mathrm{T}$ cell compartment is maintained by the proliferation of preexisting naive $\mathrm{T}$ cells. It is currently believed that exposure to pathogens over the lifetime leads to continuous attrition of naive $T$ cells with a resultant accumulation of memory cells. The depletion of ETPs and CLPs with age results in inability of the thymus to maintain thymopoiesis $(6,7)$. The generation and 
A

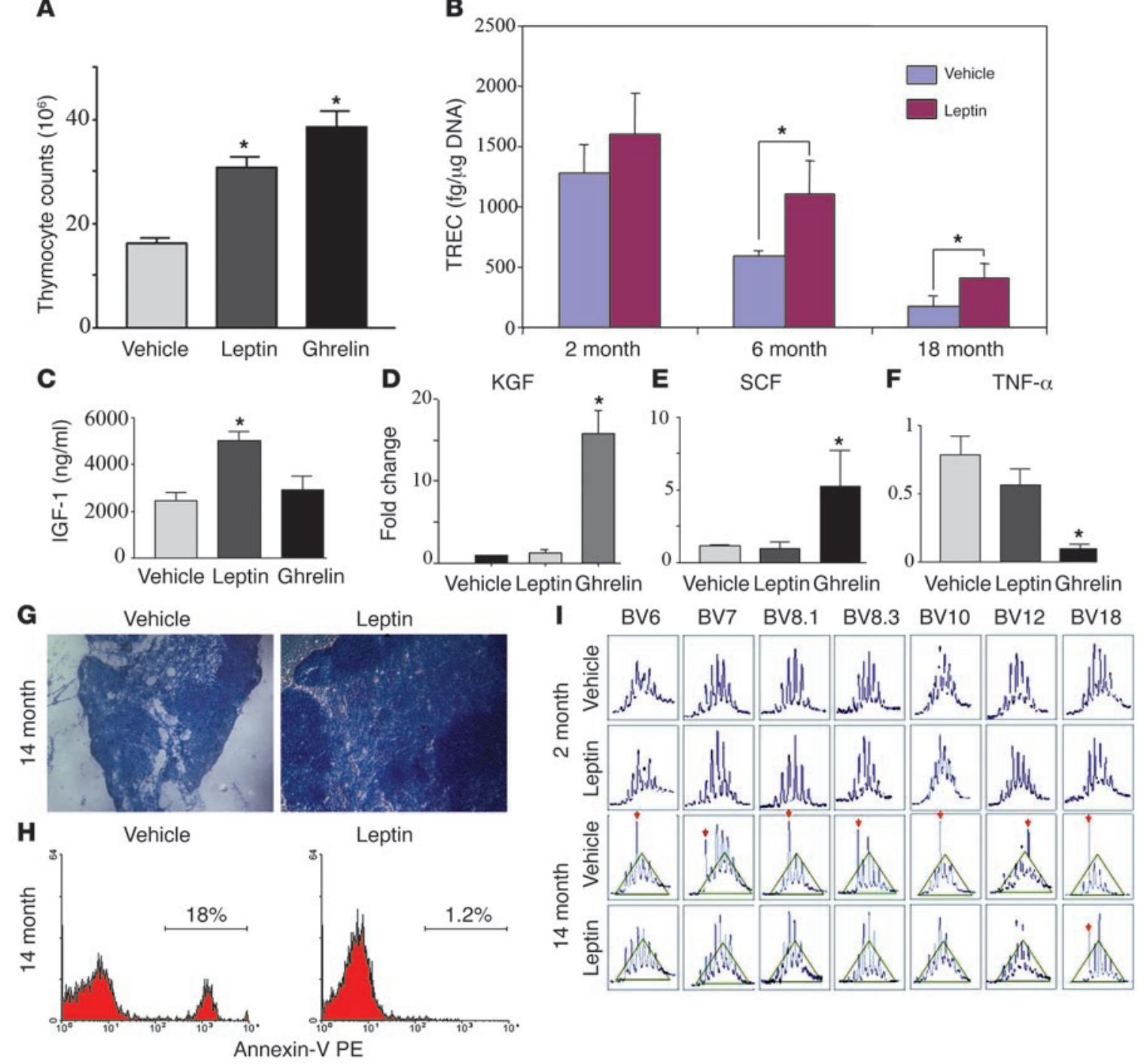

Figure 7

Leptin enhances thymopoiesis in aging mice. (A) Similar to ghrelin, leptin infusions in 14-month-old C57BL/6 mice significantly increased the total thymocyte number. (B) Leptin also enhanced the number of RTEs in aged (6- and 18-month-old) but not young (2-month-old) mice. Moreover, leptin but not ghrelin significantly increased the circulating levels of IGF-1 (C) and thymic KGF expression (D) in the thymus of aged mice. In contrast, ghrelin but not leptin significantly enhanced SCF (E) and inhibited TNF- $\alpha$ (F) mRNA expression in the aged thymus. (G) Furthermore, leptin infusion into 14-month-old BALB/c mice restored the age-related loss of cortical and medullary cellularity and the defined architecture of thymus and reduced the number of apoptotic thymocytes $(\mathbf{H})$ compared with age-matched vehicle controls. Original magnification, $\times 10$. (I) Similar to ghrelin infusion, exogenous leptin administration also increased the peripheral TCR diversity in aged (14-month-old) but not young (4-month-old) mice. ${ }^{*} P<0.01$.

maintenance of lymphoid progenitors and T cell selection, maturation, and export to the periphery is probably under the control of multiple endogenous cytokines, chemokines, and growth factors. Deficiency of IL-7 (42), for example, results in premature involution of thymus and has been hypothesized to result in decreased efficiency of maintenance of naive $\mathrm{T}$ cells. Long-term peripheral IL-7 infusions have been reported to augment total TREC numbers; however, no improvements in thymic function were observed in ref. 43. The intrathymic supplementation of IL-7 in mice also failed to increase RTE during aging (40). Furthermore, a recent study in 44 centenarians noted the presence of TREC-bearing cells in the periphery, but no difference in IL-7 or IL-7 receptor- $\alpha$ mRNA could be detected in elderly compared with young and middle-aged individuals, suggesting a limited role for IL-7 alone in strategies for thymic renewal in aging (44). A number of studies have shown that activation of somatotropic axis and ablation of gonadal steroids promote thymic rejuvenation $(24-27,30)$, clearly suggesting the resilient nature of the thymus and its capacity for renewal. We have identified that ghrelin and its receptors are expressed in the developing thymocytes and thymus and their expression decline with advancing age. While the majority of GHS-R was not expressed in TECs, partial colocalization was observed in thymic dendritic cells. The decline in ghrelin and GHS-R expression in thymic microenvironment positively correlated with reduction in thymic output. 
A

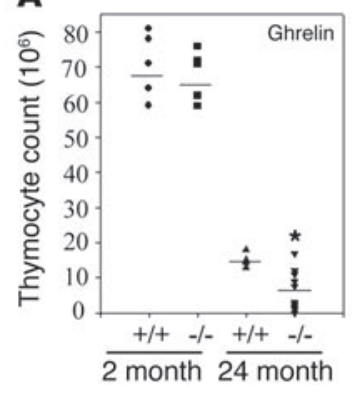

B
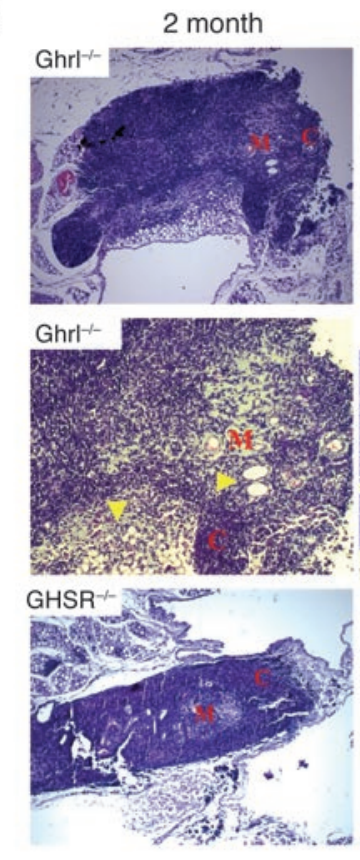
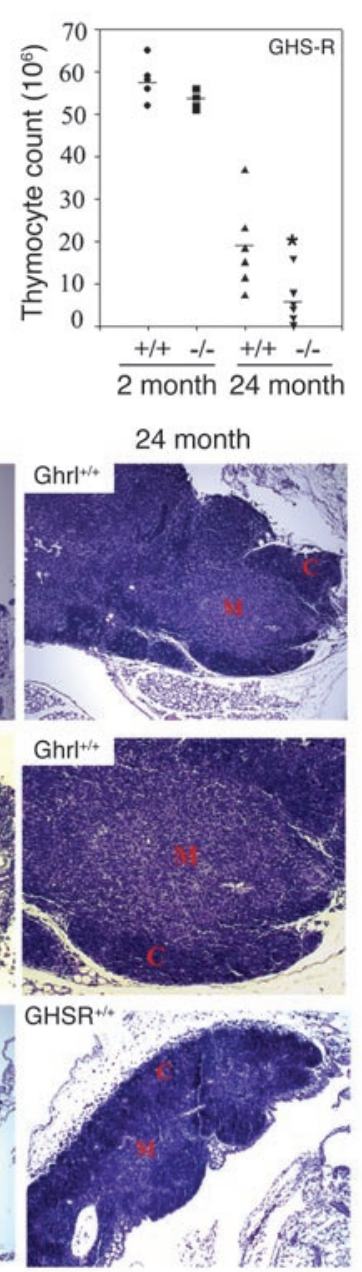

C

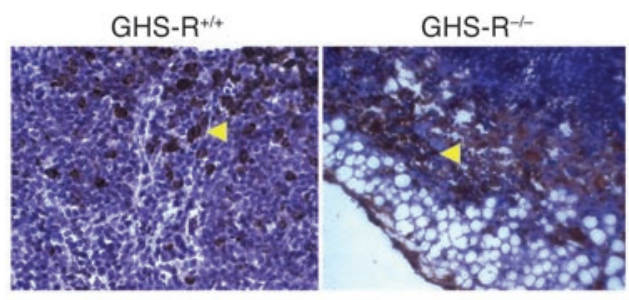

D
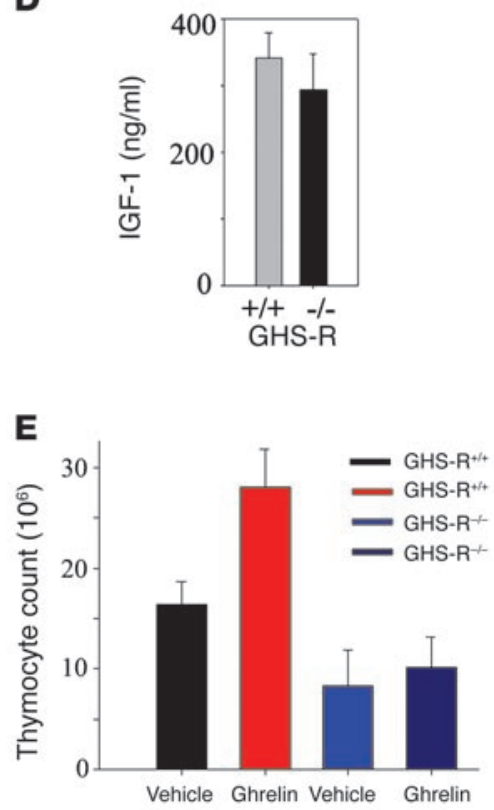

\section{Figure 8}

Ghrelin and GHS-R-deficient mice demonstrate accelerated age-associated thymic involution. (A) Thymocyte counts in ghrelin ${ }^{-/-}$and GHS-R ${ }^{-/-}$ mice did not differ at 2 months of age but were significantly reduced at 24 months of age. (B) The histological examination of thymus from 24month-old ghrelin ${ }^{-1-}$ mice revealed a greater loss of CMJs, reduced cellularity, and an increased number of adipocytes in the thymus. Original magnification, $\times 10$ (upper and lower panels); $\times 20$ (middle panels). A similar reduction in cellularity and enhanced thymic involution from agematched littermates was observed in GHS-R $\mathrm{R}^{-/}$mice. (C) $\mathrm{GHS}^{-\mathrm{R}^{-/}}$mice displayed an increase in cortical adipocyte infiltration with no significant difference in intrathymic GH expression (arrowheads). Original magnification, $\times 40$. (D) GHS-R-deficient mice did not show significant change in serum IGF-1 levels. (E) Ghrelin promoted thymopoiesis in 12-month-old mice via GHS-R-specific pathway. The 2-week-long ghrelin infusions increased the thymocyte number in WT mice but not in $\mathrm{GHSR}^{-1-}$ mice.

Such a decline in IL-7 expression with age has been reported to contribute to thymic involution (31). Given the very small size of thymus in old mice and the challenge associated with direct intrathymic delivery, we used osmotic minipumps to supplement the ghrelin in 14-month-old animals. Interestingly, ghrelin infusion led to a marked increase in thymic size, cellularity, and output in 14month-old but not in 2-month-old mice.

It has recently been suggested that TECs undergo dynamic changes throughout the life span and age-associated thymic involution might occur as a result of inability to maintain steady-state levels of TEC populations (34). Furthermore, reduced thymic output and aberrations in TEC turnover and composition that accompany the age-associated thymic involution process can be reversed by interventions that deplete endogenous sex steroid hormones $(24,30)$. We observed no significant alteration in DN,
SP, or double-positive (DP) thymic subsets following ghrelin infusions in 14-month-old animals, but we did observe significantly improved TEC and dendritic cell compartments. The changes in the thymus after ghrelin infusion appeared to affect the aging phenotype in mice and were not associated with any apparent pathology in peripheral organs or tumor development (data not shown). The lack of ghrelin-induced thymopoiesis in 2-month-old mice (when the thymic size and function were optimal) was interesting. This age-dependent increase in thymic output following ghrelin infusions raises the possibility of tight regulation of immunologic space within the thymus. Such control of organ growth and available immunologic space in an atrophied thymus might also explain the absence of thymomas in ghrelin-treated animals. Furthermore, lack of ghrelin and GHS-R in mice is not manifested in reduced thymic cellularity in young animals, suggesting a redundant role 
A
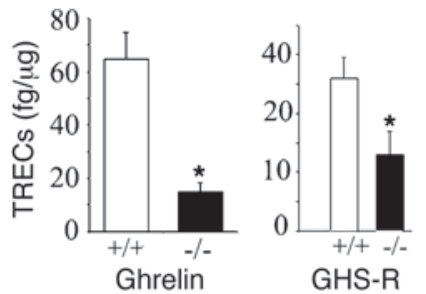

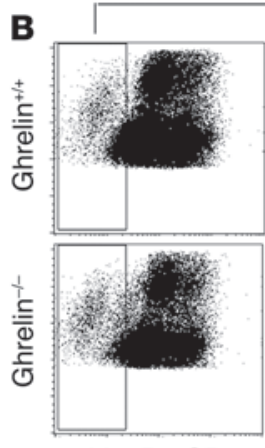

Lin-PerCP
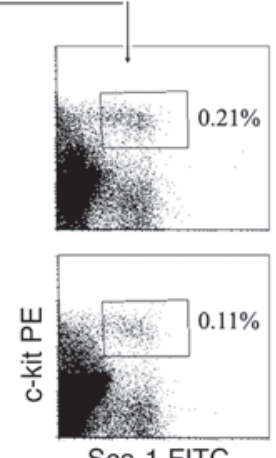

Sca-1 FITC
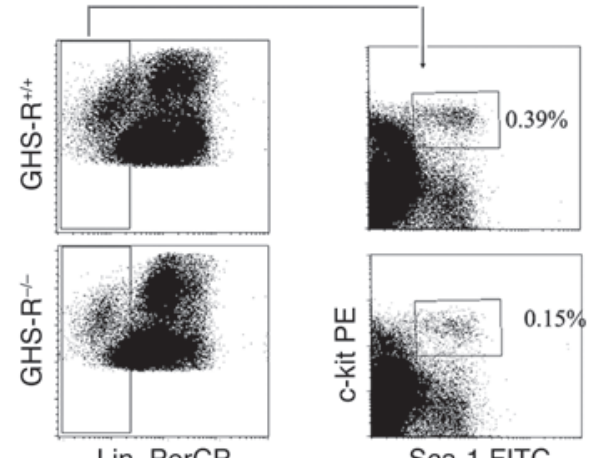

Sca-1 FITC

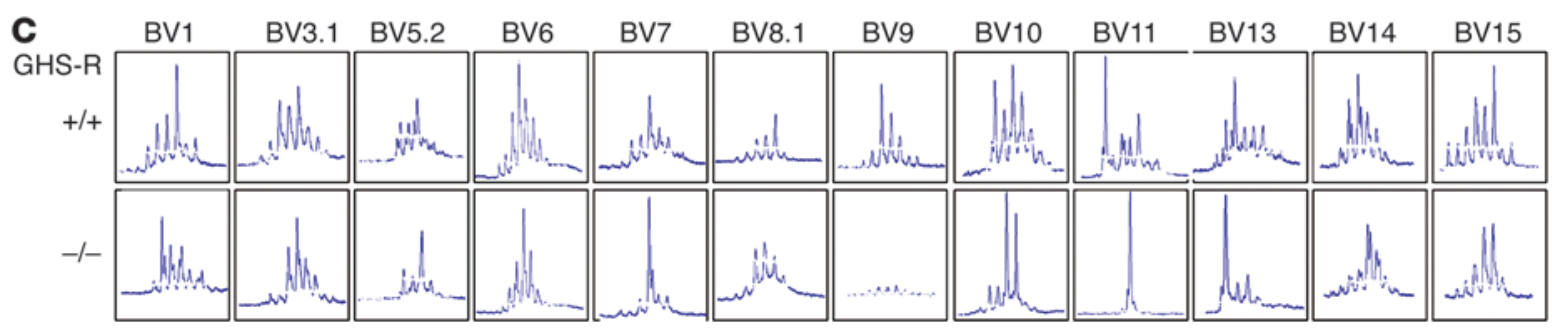

Figure 9

Lack of ghrelin signaling reduces thymopoiesis. (A) Compared with WT littermates, the 24-month-old ghrelin and GHS-R knockout mice had reduced TREC ${ }^{+}$RTEs. ${ }^{*} P<0.05$. (B) Ghrelin ${ }^{-1-}$ and GHS-R ${ }^{-1-}$ mice demonstrated a marked decrease in LSK in bone marrow. GHS-R-deficient mice show a marked decline in TCR repertoire in peripheral CD4+ cells with age. (C) CDR3 size spectratyping was used to analyze the TCR repertoire. Total RNA from splenic CD4+ cells was reverse transcribed and amplified by PCR with 23 pairs of TCR-BV- and FAM-labeled C $\beta$-specific primers. Results are shown for $V \beta$ families showing major perturbation as a density peak histogram. CDR3 sizes are shown on the $x$ axis, and peak fluorescence intensity is shown on the $y$ axis. Results are representative of 4 experiments. The V $\beta 7,-11,-13$, and -19 of GHS-R ${ }^{-/-}$mice displayed a markedly monoclonal spectratype (marked increase in the intensity of single peak/band), while V $\beta 1,-3.1,-5.2,-6,-8.1,-10,-14$, and -15 showed a typical oligoclonal pattern and distortion from Gaussian distribution. Interestingly, none of GHS-R ${ }^{-1-}$ mice had amplification of V $\beta 9$ compared with WT littermates.

for this ligand-receptor pair in thymus or possibly redundant signaling pathways compensating for the ghrelin/GHS-R loss in the knockout mice. However, as these animals age physiologically, a markedly accelerated thymic involution phenotype was observed. Similarly, the 2-month-old IL-12 knockout mice did not exhibit any apparent defects in the thymic structure or T cell development but did display an accelerated thymic involution phenotype as they got older (45). Ghrelin is a potent endogenous stimulator of GH secretion (8), and activation of GH-IGF-1 is known to augment thymopoiesis $(25,26)$. However, we did not observe a significant difference in peripheral IGF-1 levels after ghrelin infusion in 14-month-old vehicle-infused mice or in ghrelin-/- mice that displayed increased age-associated thymic involution. IGF-1 is commonly utilized as a surrogate marker for changes in circulating $\mathrm{GH}$ levels. Moreover, given that GH release in blood is pulsatile in nature, the determination of GH by a single blood draw in mice is unreliable. Therefore, we examined the GH expression directly in the pituitary of ghrelin-treated 14-month-old mice and observed a greater number of $\mathrm{GH}^{+}$cells following ghrelin infusion. These data suggest that there might be an uncoupling of the ghrelin-IGF-1 axis in old animals and a ghrelin-induced increase in thymic output in aging may be independent of GH and/or IGF-1.

The thymic output and emergence of RTEs in the periphery can be assessed by quantitation of TRECs, which are episomal DNA fragments released in naive $T$ cells as a result of rearrangement of TCR. We observed that exogenous ghrelin infusion in old mice increased the peripheral TREC levels by greater than 2 -fold, and the old ghrelin- and GHS-R-deficient mice displayed a greater loss of TREC-positive T cells. These data strongly support a physiological role for ghrelin in thymopoiesis.

An optimum TCR diversity of naive T cells is critical for the ability of the host to mount successful immune responses against various foreign pathogens while avoiding reaction against the self antigens (36). Other than physiological aging, contraction of the TCR repertoire is commonly observed in patients with SCID, HIV infection, and various cancers and those who have undergone bone marrow transplantation, and currently no endogenous growth factors or cytokines have been identified that exert a positive impact on broadening the spectrum of TCR during aging. We observed that ghrelin improved the TCR diversity by improving the $\mathrm{V} \beta 8.1,-8.2$, and -8.3 in peripheral $\mathrm{CD}^{+}$and $\mathrm{CD}^{+}$cells. In addition, CDR3 length analysis revealed an enhancement of $\mathrm{V} \beta 4,-16$, and -19 . The exact functional relevance of this improvement of TCR repertoire during aging remains to be ascertained, but previous studies have suggested that resolution of MOPC315 tumors in mice is critically dependent on V $\beta 8.1,-8.2$, and -8.3 (46). Ghrelin has been proposed as a possible therapeutic against cancer-associated cachexia. However, the direct effects of ghrelin on cancer require further investigation, as some of our recent data has suggested that ghrelin may promote astrocytoma cell growth, migration, and invasion in vitro (47).

Previous studies have demonstrated that in humans with SCID, the HLA- or haploidentical T cell-depleted allogeneic bone marrow transplantation results in a marked increase in TREC values 
in recipients with a concomitant enhancement of peripheral TCR diversity (48). Similarly, the 24-month-old GHS-R knockout mice that had reduced RTEs displayed marked perturbation in TCR spectratype of peripheral $\mathrm{CD}^{+} \mathrm{T}$ cells with monoclonal or oligoclonal expansion and also showed skewing from a more Gaussian distribution of $V \beta$-specific peaks. Most strikingly, we did not detect V $\beta$ 9-specific peaks in the TCR repertoire of 24-month-old GHS-R knockout mice compared with littermate controls. It has previously been reported that the protozoal parasite Plasmodium yoelii in murine models of malaria can cause specific deletion of $\mathrm{V} \beta 9$ possibly due to the superantigenic activity due to sporozoites or erythrocytic stage of Plasmodium (49). The strains of mice lacking V $\beta 9$ were more susceptible to infection with a greater duration and peak of parasitemia (49). Based on our findings, it seems feasible that deficiency of GHS-R signaling might have a severe impact on the clearance of intracellular pathogens and further deteriorate the capability of the immune system to respond to antigenic challenge during aging. Conversely, the pharmacological activation of signals emanating from ghrelin receptor could facilitate enhancing immunity during various conditions associated with immune deficiency.

In order to study the possible mechanisms behind the effects of ghrelin on thymic renewal, we analyzed the hematopoietic progenitors with lymphoid potential in the thymus as well as bone marrow. It has been reported that loss of ETPs and reduced homing of bone marrow progenitors to thymus results in progressive reduction of newly formed naive $T$ cells from the thymus with age (7). Ghrelin significantly increased the ETPs in the DN1 compartment without significantly affecting other stages of $\mathrm{T}$ cell development. The augmentation of ETPs in the aging thymus after ghrelin infusion was also associated with an increased percentage of LSK cells in the bone marrow. Furthermore, a physiological role for ghrelin and GHS-R in maintenance of hematopoietic cell progenitors was evident, as 24-month-old mice deficient in ghrelin as well as GHS-R 0exhibited a reduced number of LSK cells in the bone marrow. An age-associated reduction in LSK cells from BM niches and the resultant unavailability of $\mathrm{T}$ cell progenitors may lead to a loss of thymopoiesis over time. Lack of an overt phenotype in young ghrelin and GHS-R knockout mice has previously been noted $(50,51)$; however, recent studies indicate that exposing these mice to the metabolic stress of high-fat and high-sugar diets results in resistance to weight gain and obesity $(52,53)$. Our data suggest that during physiological aging, lack of ghrelin signaling leads to an accelerated thymic involution.

Given that leptin is known to be a proinflammatory hormone and that ghrelin and leptin exert reciprocal regulatory effects on metabolism and cytokine expression $(12,13)$, we have further investigated whether such mechanisms might also exist within the aging thymus. While ghrelin infusion did not appear to significantly affect circulating leptin levels or thymic leptin mRNA levels (data not shown), leptin infusion into aged but not young mice did significantly enhance thymopoiesis. Furthermore, unlike ghrelin, leptin infusion resulted in a marked increase in thymic KGF mRNA expression with little effect on TNF- $\alpha$ or SCF mRNA expression. Recent work has demonstrated that exogenous KGF administration can significantly abrogate age-associated thymic involution (54). Thus it might be feasible that the prothymic effects of leptin may be related to its effects on thymic KGF expression. In addition, given that leptin can also promote thymic output in an LPS model of endotoxemia (33) and our current data that leptin significantly attenuates age-related thymic involution, the ability of leptin to promote inflammation (12) may not be a critical factor in thymic renewal. Our data suggest that leptin and ghrelin may operate through distinct pathways to regulate thymopoiesis and thymic restoration in aged mice.

These findings describe a previously unrecognized function for ghrelin in thymic biology and aging and suggest that this orexigenic peptide hormone may play an important role in coupling energy balance to $\mathrm{T}$ cell lymphopoiesis. Interplay between ghrelin and ghrelin receptor interactions in the thymic compartment and bone marrow stem cell niches leads to rejuvenation of thymic output and improved T cell repertoire in old animals. Given ghrelin's potent orexigenic effects, several pharmacological (55) and vaccine-based strategies (56) to disrupt ghrelin signaling have been proposed as an intervention against obesity. However, additional detailed studies in rodent or primate models are warranted, as long-term neutralization of already suboptimal ghrelin signaling in obesity might be fraught with potentially harmful effects on the thymic output and immune responsiveness to antigenic challenge. Ghrelin inhibits inflammation $(12,16)$, increases $\mathrm{GH}$ and appetite, and promotes learning and memory (57) and is therefore proposed as a hormone of significant therapeutic benefit (58). With identification of ghrelin's thymopoietic effects during aging, we propose that harnessing the ghrelin-GHS-R pathway might significantly benefit the aged and immunocompromised host in promoting $\mathrm{T}$ cell output and TCR repertoire.

\section{Methods}

Mice and in vivo treatments. Balb/c mice were maintained under the specific pathogen-free conditions of the National Institute on Aging (NIA) animal facility using protocols approved by the animal care and use committee of the NIA Intramural Research Program located in Baltimore, Maryland, USA. We utilized 2-, 14-, 20- and 24-month-old Balb/c and 8-month-old female C57BL/ 6 mice for ghrelin infusions using osmotic minipumps. The osmotic Alzet minipumps (Durect) were prepared according to the manufacturer's recommendations, and pumps containing ghrelin or vehicle were implanted s.c. under xylazine/ketamine anesthesia. Ghrelin was infused at a concentration of $1.25 \mu \mathrm{g} / \mathrm{h}$ for 2 weeks, and control mice were implanted with osmotic minipumps containing the PBS vehicle alone. Recombinant mouse leptin (Sigma-Aldrich) was infused at a dose of $292.2 \mathrm{ng} / \mathrm{h}$ for 2 weeks using s.c. osmotic minipumps in 2-, 6-, and 18-month-old $\mathrm{C} 57 \mathrm{BL} / 6$ mice and 4- and 14-month-old Balb/c female mice. All mice were purchased from the NIA aging rodent colony.

Generation of the ghrelin $/$ - and GHS-R $/$ - mice has been previously described $(50,51)$. Ghrelin and GHS-R knockout mice and WT littermates were aged for 12 to 24 months at the Baylor College of Medicine, and subsequent experiments were performed on these mice after providing at least 3-4 days of rest upon arrival at the NIA animal facility. A group of GHS- $\mathrm{R}^{-1-}$ mice and WT littermate control mice were also infused with ghrelin in order to study the specificity of ghrelin's thymic effects. Moreover, female Balb/c mice at 2, 4, 12, 14, 18, and 24 months of age were utilized to generate the aging thymus tissue sections shown in Figure 1.

Cell enrichment, antibodies, flow cytometry, and cell sorting. Peripheral T cells were isolated from single cell suspensions of splenocytes using mouse $T$ cell enrichment columns (R\&D Biosystems) according to the manufacturer's instructions. Thymocytes, splenocytes, bone marrow, and peripheral blood mononuclear cells were immunolabeled with optimal dilutions of FITC-, PE-, APC- or PerCP-conjugated antibodies. The thymocytes were labeled with CD4-FITC and CD8-PE and purified for DP, SP, and DN thymocyte subsets using flow sorter (MoFlow Cytomation; DAKO). We studied DN1 
cells for the ETP and CLP population by employing a dump gating scheme as described previously $(6,7,41)$. Briefly, to identify ETPs, thymocytes were labeled for Lin ${ }^{+}$cells by utilizing PE-conjugated anti-CD11b, -Gr-1, -CD45R, -CD3, -CD8, - $\alpha \beta$ TCR, - $\gamma \delta$ TCR, -pan-NK, -NK1.1, -CD11c, -CD19, -Ter119, and -CD127 antibodies. The PE-labeled Lin- cells lacking CD25 and expressing c-Kit were designated ETPs as previously described (41). The BM and blood LSK populations were identified as previously described (6).

Real-time RT-PCR. Total RNA was isolated from cells using RNeasy mini columns (QIAGEN). The cDNA synthesis and RT-PCR was performed as described previously (12).

IGF-1 assay. Mouse serum was diluted 500-fold, and the levels of circulating IGF-1 were analyzed by ELISA (R\&D Systems) according to the manufacturer's instructions.

Quantification of signal-joint TRECs. DNA was isolated from purified splenic T cells using DNA purification kit (Gentra Systems). PCR was performed

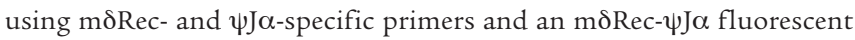
probe as described previously $(35,43)$. The standard curves for murine TRECs were generated using a $\delta$ Rec- $\psi \mathrm{J} \alpha$ TREC PCR product cloned into a PCR-XL-TOPO plasmid, a generous gift from Yu-Waye Chu (National Cancer Institute, NIH, Bethesda, Maryland, USA).

Immunohistochemistry. For GHS-R and ghrelin staining of thymic sections, paraffin-embedded thymi were sectioned and deparaffinized using xylene, $100 \% \mathrm{ETOH}, 95 \% \mathrm{ETOH}, 75 \% \mathrm{ETOH}$, and $\mathrm{H}_{2} \mathrm{O}$ series, and antigens were retrieved by steaming samples in DAKO target retrieval buffer (DAKO) for 20 minutes. After blocking the nonspecific binding sites, sections were incubated overnight at $4^{\circ} \mathrm{C}$ with appropriate antibodies (Phoenix Pharmaceuticals). Sections were stained with 3,3'-diaminobenzidine substrate and counterstained with Meyer hematoxylin and eosin. Negative controls generated by the exclusion of the primary antibody and through the use of an unrelated isotype-matched IgG failed to demonstrate any specific labeling. In addition, for the visualization of lipid droplet and adipocyte populations, thymi were flash frozen and subsequently embedded in Stephens Scientific Frozen Section Medium and cut into 5 - $\mu \mathrm{m}$-thick cryostat sections using the Microm HM 500 OM microtome. At least 3 serial sections were utilized for each staining. Tissue sections were then fixed with $3.7 \%$ paraformaldehyde and stained with Oil Red $\mathrm{O}$ to visualize lipid droplets in cells and regions in the thymic tissue.

V $\beta$ TCR spectratyping analysis. The analysis of hypervariable CDR3 of $\beta$ chain offers a practical approach for the global qualitative assessment of diversity of TCR repertoire. For TCR spectratyping and CDR3 length PCR analysis, a FAM-labeled nested constant $\beta$-region primer was used in combination with 24 multiplexed forward murine V $\beta$-specific primers. PCR was performed for 35 cycles with denaturation at $94^{\circ} \mathrm{C}$ for 30 seconds, annealing for $55^{\circ} \mathrm{C}$ for 30 seconds and 1 minute extension at $72^{\circ} \mathrm{C}$, and the PCR products were analyzed on ABI3100 genetic analyzer (Applied Biosystems). Each V $\beta$-J $\beta$ rearrangement was visualized by $6-8$ bands, and each band or peak represented 1 or a set of $\mathrm{T}$ cell clones bearing the same CDR3 length. A typical polyclonal repertoire for a specific $V \beta$ family was represented by $6-8$ peaks arranged in a Gaussian profile with the highest intensity CDR3 segment in the center.

Statistics. The results are expressed as the mean \pm SEM. The differences between the means and the effects of treatments were determined by 1 -way ANOVA using Tukey's test, which protects the significance $(P<0.05)$ of all pair combinations.

\section{Acknowledgments}

We would like to thank Arnell Carter, Dorothy Bertak, and Ana Lustig for expert technical assistance and Valeria deMelloCoelho for preparation of the thymic tissue arrays in our laboratory. We would also like to thank Christa Morris, Joe Chrest, and Robert Wersto within the NIA Flow Cytometry Facility for cell sorting and sample analysis as well as the staff of the Comparative Medicine Section of the NIA animal facility for their support. We would also like to thank Ryo Matoba and Ramaiah Nagaraja in the NIA Laboratory of Genetics for their help with ABI3100 genetic analyzer. We would also like to thank Dan L. Longo, Ranjan Sen, Nan-ping Weng, Jyoti M. Sen, and P. Ghosh at the NIA for helpful discussions and reviewing this manuscript. This research was supported in part by the Intramural Research Program of the NIA and NIH grants R01 AG19230-01 and R01 AG18895-02 to R.G. Smith.

Received for publication September 5, 2006, and accepted in revised form June 26, 2007.

Address correspondence to: Dennis D. Taub, Clinical Immunology Section, Laboratory of Immunology, National Institute on Aging, Intramural Research Program, NIH, 5600 Nathan Shock Drive, Baltimore, Maryland 21224, USA. Phone: (410) 558-8159; Fax: (410) 558-8284; E-mail: taubd@grc.nia.nih.gov. Or to: Vishwa Deep Dixit, Laboratory of Neuroendocrine Immunology, Pennington Biomedical Research Center, Louisiana State University, 6400 Perkins Road, Baton Rouge, Louisiana 70808, USA. Phone: (225) 763-2719; Fax: (225) 763-0261; E-mail: vishwa.dixit@pbrc.edu.
1. Taub, D.D., and Longo, D.L. 2005. Insights into thymic aging and regeneration. Immunol. Rev. 205:72-93.

2. Linton, P.E., and Dorshkind, K. 2004. Age related changes in lymphocyte development and function. Nat. Immunol. 5:133-139.

3. Haynes, L., Eaton, S.M., Burns, E.M., Randall, T.D., and Swain, S.L. 2005. Newly generated CD4 T cells in aged animals do not exhibit age-related defects in response to antigen. J. Exp. Med. 201:845-851.

4. Weng, N.P. 2006. Aging of the immune system: how much can the adaptive immune system adapt? Immunity. 24:495-499.

5. Hale, J.S., Boursalian, T.E., Turk, G.L., and Fink, P.J. 2006. Thymic output in aged mice. Proc. Natl. Acad. Sci.U.S. A. 103:8447-8452.

6. Bhandoola, A., and Sambandam, A. 2006. From stem cell to T cell: one route or many? Nat. Rev. Immunol. 6:117-126.

7. Min, H., Montecino-Rodriguez, E., and Dorshkind, K. 2004. Reduction in the developmental potential of intrathymic $\mathrm{T}$ cell progenitors with age. J. Immunol.
173:245-250

8. Kojima, M., et al. 1999. Ghrelin is a growth-hormone- releasing acylated peptide from stomach. Nature. 402:656-660.

9. Smith, R.G. 2005. Development of growth hormone secretagogue. Endocr. Rev. 26:346-360.

10. Kojima, M., and Kengawa, K. 2005. Ghrelin: structure and function. Physiol. Rev. 85:495-522.

11. Gnanapavan, S., et al. 2002. The tissue distribution of the mRNA of ghrelin and subtypes of its receptor, GHS-R, in humans. J. Clin. Endocrinol. Metab. 87:2988-2991.

12. Dixit, V.D., et al. 2004. Ghrelin inhibits leptin- and activation-induced proinflammatory cytokine expression by human monocytes and T cells. J. Clin. Invest. 114:57-66. doi:10.1172/JCI200421134.

13. Dixit, V.D., and Taub, D.D. 2005. Ghrelin and immunity: a young player in an old field. Exp. Gerontol. 40:900-910.

14. Li, W.G., et al. 2004. Ghrelin inhibits proinflammatory responses and nuclear factor-kappaB activation in human endothelial cells. Circulation.
109:2221-2226.

15. Granado, M., Priego, T., Martin, A.I., Villanua, M.A., and Lopez-Calderon, A. 2005. Anti-inflammatory effect of the ghrelin agonist growth hormonereleasing peptide-2 (GHRP-2) in arthritic rats. Am. J. Physiol. Endocrinol. Metab. 288:E486-E492.

16. Gonzalez-Rey, E., Chorny, A., and Delgado, M. 2006. Therapeutic action of ghrelin in a mouse model of colitis. Gastroenterology. 130:1707-1720.

17. Chang, L., et al. 2005. Therapeutic effects of ghrelin on endotoxic shock in rats. Eur. J. Pharmacol. 473:171-176.

18. Sempowski, G.D., et al. 2000. Leukemia inhibitory factor, oncostatin M, IL-6 and stem cell factor mRNA expression in human thymus increases with age and are associated with thymic atrophy. J. Immunol. 164:2180-2187.

19. van den Brink, M.R., Alpdogan, O., and Boyd, R.L. 2004. Strategies to enhance T-cell reconstitution in immunocompromised patients. Nat. Rev. Immunol. 4:856-867.

20. Hakim, F.T., et al. 2005. Age-dependent incidence, 
time course, and consequences of thymic renewal in adults. J. Clin. Invest. 115:930-939. doi:10.1172/ JCI200522492

21. Heng, T.S., et al. 2005. Effects of castration on thymocyte development in two different models of thymic involution. J. Immunol. 175:2982-2993.

22. Marchetti, B., et al. 1989. Luteinizing hormonereleasing hormone (LHRH) agonist restoration of age-associated decline of thymus weight, thymic LHRH receptors, and thymocyte proliferative capacity. Endocrinology. 125:1037-1045.

23. Dixit, V.D., Sridaran, R., Edmonsond, M.A., Taub, D., and Thompson, W.E. 2003. Gonadotropinreleasing hormone attenuates pregnancy-associated thymic involution and modulates the expression of antiproliferative gene product prohibitin. Endocrinology. 144:1496-1505.

24. Sutherland, J.S., et al. 2005. Activation of thymic regeneration in mice and humans following androgen blockade. J. Immunol. 175:2741-2753.

25. Murphy, W.J., Durum, S.K., and Longo, D.L. 1992. Role of neuroendocrine hormones in murine T cell development. Growth hormone exerts thymopoietic effects in vivo. J. Immunol. 149:3851-3857.

26. French, R., et al. 2002. Age-associated loss of bone marrow hematopoietic cells is reversed by GH and accompanies thymic reconstitution. Endocrinology. 143:690-699.

27. Montecino-Rodriguez, E., Clark, R., and Dorshkind, K. 1998. Effects of insulin-like growth factor administration and bone marrow transplantation on thymopoiesis in aged mice. Endocrinology. 139:4120-4126.

28. Koo, G.C., et al. 2001. Immune enhancing effect of a growth hormone secretagogue. J. Immunol. 166:4195-4201.

29. Akiyama, T., et al. 2005. Dependence of self-tolerance on TRAF6-directed development of thymic stroma. Science. 308:248-251.

30. Gray, D.H., et al. 2006. Developmental kinetics, turnover, and stimulatory capacity of thymic epithelial cells. Blood. 108:3777-3785

31. Andrew, D., and Aspinall, R. 2002. Age-associated thymic atrophy is linked to a decline in IL-7 production. Exp. Gerontol. 37:455-463.

32. Howard, J.K., et al. 1999. Leptin protects mice from starvation-induced lymphoid atrophy and increas- es thymic cellularity in ob/ob mice. J. Clin. Invest. 104:1051-1059.

33. Hick, R.W., et al. 2006. Leptin selectively augments thymopoiesis in leptin deficiency and lipopolysaccharide-induced thymic atrophy. J. Immunol. 177:169-176.

34. Gray, D.H., et al. 2005. Controlling the thymic microenvironment. Curr. Opin. Immunol. 17:137-143.

35. Douek, D.C., et al. 1998. Changes in the thymic function with age and during the treatment of HIV infection. Nature. 396:690-695.

36. Nikolich-Zugich, J., Slifka, M.A., and Messaoudi, I. 2004. The many important facets of T-cell repertoire diversity. Nat. Rev. Immunol. 4:123-132.

37. Naylor, K., et al. 2005. The influence of age on $\mathrm{T}$ cell generation and TCR diversity. J. Immunol. 174:7446-7452.

38. Lind, E.F., Prockop, S.E., Porritt, H.E., and Petrie, H.T. 2001. Mapping precursor movement through the postnatal thymus reveals specific microenvironments supporting defined stages of early lymphoid development. J. Exp. Med. 194:127-134.

39. Zuniga-Pflucker, J.C. 2004. T-cell development made simple. Nat. Rev. Immunol. 4:67-72.

40. Phillips, J.A., et al. 2004. IL-7 gene therapy in aging restores early thymopoiesis without reversing involution. J. Immunol. 173:4867-4874.

41. Bhandoola, A., and Sambandam, A. 2006. From stem cell to T cell: one route or many? Nat. Rev. Immunol. 6:117-126.

42. von Freeden-Jeffry, U., et al. 1995. Lymphopenia in interleukin (IL)-7 gene-deleted mice identifies IL-7 as a nonredundant cytokine. J. Exp. Med. 181:1519-1525.

43. Chu, Y.W., et al. 2004. Exogenous IL-7 increases recent thymic emigrants in peripheral lymphoid tissue without enhanced thymic function. Blood. 104:1110-1119.

44. Nasi, M., et al. 2006. Thymic output and functionality of the IL-7/IL-7 receptor system in centenarians: implications for the neolymphogenesis at the limit of human life. Aging Cell. 5:167-175.

45. Li, L., et al. 2004. IL-12 inhibits thymic involution by enhancing IL-7- and IL-2-induced thymocyte proliferation. J. Immunol. 172:2909-2916.

46. Mokyr, M.B., Prokhorova, A., Rubin, M., and Blue- stone, J.A. 1994. Insight into the mechanism of TCR-V beta 8+/CD8+ T cell-mediated MOPC-315 tumor eradication. J. Immunol. 153:3123-3134.

47. Dixit, V.D., et al. 2006. Ghrelin and the growth hormone secretagogue receptor constitute a novel autocrine pathway in astrocytoma motility. J. Biol. Chem. 281:16681-16690.

48. Sarzotti, M., et al. 2003. T cell repertoire development in humans with SCID after nonablative allogeneic marrow transplantation. J. Immunol. 170:2711-2718

49. Pied, S., et al. 1997. Evidence for superantigenic activity during murine malaria infection. Int. Immunol. 9:17-25.

50. Sun, Y., Ahmed, S, and Smith, R.G. 2003. Deletion of ghrelin impairs neither growth nor appetite. Mol. Cell. Biol. 23:7973-7981.

51. Sun, Y., Wang, P., Zheng, H., and Smith, R.G. 2004 Ghrelin stimulation of growth hormone release and appetite is mediated through the growth hormone secretagogue receptor. Proc. Natl. Acad. Sci. U. S. A. 101:4679-4684.

52. Zigman, J.M., et al. 2005. Mice lacking ghrelin receptors resist the development of diet-induced obesity. J. Clin. Invest. 115:3564-3572. doi:10.1172/ JCI26002.

53. Wortley, K.E., et al. 2005. Absence of ghrelin protects against early-onset obesity. J. Clin. Invest. 115:3573-3578. doi:10.1172/JCI26003.

54. Min, D., et al. 2007. Sustained thymopoiesis and improvement in functional immunity induced by exogenous KGF administration in murine models of aging. Blood. 109:2529-2537.

55. Zhao, H., and Liu, G. 2006. Growth hormone secretagogue receptor antagonists as anti-obesity therapies? Still an open question. Curr. Opin. Drug Discov. Devel. 9:509-515.

56. Zorrilla, E.P., et al. 2006. Vaccination against weight gain. Proc. Natl. Acad. Sci. U. S. A. 103:13226-13231.

57. Diano, S., et al. 2006. Ghrelin controls hippocampal spine synapse density and memory performance. Nat. Neurosci. 9:381-388.

58. Kojima, M., and Kangawa, K. 2006. Drug insight: the functions of ghrelin and its potential as a multitherapeutic hormone. Nat. Clin. Pract. Endocrinol. Metab. 2:80-88. 